\section{(1) \\ CrossMark}

\title{
The impact of digital health technologies on tuberculosis treatment: a systematic review
}

\author{
Brian Kermu Ngwatu ${ }^{1,4}$, Ntwali Placide Nsengiyumva ${ }^{2,4}$, Olivia Oxlade ${ }^{2}$, \\ Benjamin Mappin-Kasirer ${ }^{2}$, Nhat Linh Nguyen ${ }^{1}$, Ernesto Jaramillo ${ }^{1}$, \\ Dennis Falzon ${ }^{1}$ and Kevin Schwartzman ${ }^{2}$ on behalf of the Collaborative group \\ on the impact of digital technologies on $\mathrm{TB}^{3}$
}

\begin{abstract}
Affiliations: ${ }^{1}$ Global TB Programme, World Health Organization, Geneva, Switzerland. ${ }^{2}$ Montreal Chest Institute, Respiratory Epidemiology and Clinical Research Unit, Research Institute of the McGill University Health Centre, and McGill International Tuberculosis Centre, Montreal, Canada. ${ }^{3}$ For a full list of the collaborative group see the Acknowledgements. ${ }^{4}$ Both authors contributed equally.
\end{abstract}

Correspondence: Kevin Schwartzman, Respiratory Epidemiology and Clinical Research Unit, Montreal Chest Institute - Glen D5-2511, McGill University Health Centre, 1001 boul Decarie, Montreal QC, Canada H4A 3J1. E-mail: kevin.schwartzmandamcgill.ca

@ERSpublications

Digital technologies are being used to support TB treatment adherence, but evidence for their impact needs to improve http://ow.ly/1riC30h6hu3

Cite this article as: Ngwatu BK, Nsengiyumva NP, Oxlade O, et al. The impact of digital health technologies on tuberculosis treatment: a systematic review. Eur Respir J 2018; 51: 1701596 [https://doi.org/ 10.1183/13993003.01596-2017].

ABSTRACT Digital technologies are increasingly harnessed to support treatment of persons with tuberculosis (TB). Since in-person directly observed treatment (DOT) can be resource intensive and challenging to implement, these technologies may have the potential to improve adherence and clinical outcomes. We reviewed the effect of these technologies on TB treatment adherence and patient outcomes.

We searched several bibliographical databases for studies reporting the effect of digital interventions, including short message service (SMS), video-observed therapy (VOT) and medication monitors (MMs), to support treatment for active TB. Only studies with a control group and which reported effect estimates were included.

Four trials showed no statistically significant effect on treatment completion when SMS was added to standard care. Two observational studies of VOT reported comparable treatment completion rates when compared with in-person DOT. MMs increased the probability of cure (RR 2.3, 95\% CI 1.6-3.4) in one observational study, and one trial reported a statistically significant reduction in missed treatment doses relative to standard care (adjusted means ratio 0.58 , 95\% CI $0.42-0.79$ ).

Evidence of the effect of digital technologies to improve TB care remains limited. More studies of better quality are needed to determine how such technologies can enhance programme performance.

This article has supplementary material available from erj.ersjournals.com

Received: Aug 042017 | Accepted after revision: Oct 062017

Support statement: This work was funded by the European Respiratory Society, via the World Health Organization Stop TB Programme. Funding information for this article has been deposited in the Crossref Funder Registry

Conflict of interest: Disclosures can be found alongside this article at erj.ersjournals.com

This is one of a selection of articles published as ERJ Open papers, as part of an initiative agreed between the European Respiratory Society and the World Health Organization.

Copyright OERS 2018. This version is distributed under the terms of the Creative Commons Attribution NonCommercial Licence 4.0. 


\section{Introduction}

Digital technologies are changing healthcare delivery globally, as witnessed by the dramatic growth in such areas as electronic health records, telehealth for "virtual" patient encounters, and teleradiology for remote interpretation of imaging studies [1]. There is also increasing recognition that digital technologies can support medication adherence. Examples include the use of video phone calls for observation of medication ingestion, and for live discussion of any problems or concerns; the use of short message service ((SMS), i.e. text messaging) for ongoing communication between patients and providers; and the use of SMS or electronic medication monitors (MMs) for automatic reminders, and/or for real-time monitoring of medication self-administration which is then fed back to providers. MMs most commonly take the form of "smart" pill bottles, which can keep track of pill counts and bottle openings, and transmit adherence reports to treating health professionals [2-4].

Digital treatment support can lead to better treatment results in patients with chronic diseases, such as diabetes [5-7]. As with tuberculosis (TB), these chronic conditions require long-term commitment to treatment by patients and caregivers. Several systematic reviews aimed at measuring the effectiveness of mobile communication technologies, such as SMS, have also documented improved adherence to antiretroviral treatment [8], and higher rates of smoking cessation [9]. Other studies evaluating SMS-based interventions in maternal-child health services [10,11] found improved contraceptive pill adherence [12] and higher proportions of deliveries by trained personnel [13].

Suboptimal adherence to TB treatment is common, with a global treatment success rate of $75 \%$ for new and relapse TB cases in 2014 [14], despite long-standing support for strategies such as direct observation to help patients complete their treatment[15]. While barriers to adherence have been much researched, there has been little progress in lessening treatment burden by mitigating frequent adverse reactions, or substantially shortening the duration of current regimens; minimum 6 months for drug-susceptible TB (DS-TB) and typically 18 months or more for rifampicin-resistant TB (RR-TB). Globally, the treatment success rate for RR-TB is approximately 50\%; about one in seven RR-TB patients is lost to follow-up during treatment [14]. It is highly relevant to identify which programmatic solutions could contribute to improved TB treatment adherence, and ultimately lead to better patient outcomes.

For example, enhanced communication via SMS or video calls could strengthen relationships between patients, families and healthcare providers, and promote retention in care as well as treatment adherence. Early detection of missed treatment doses (e.g. by electronic MMs) could allow healthcare workers to address any patient concerns or barriers, to suggest specific steps to improve adherence, and to mitigate potential loss to follow-up. Similarly, early signalling to healthcare providers of potential drug side-effects could allow for treatment changes and/or effective symptomatic management.

The use of digital health applications to improve treatment support for active TB patients also appeals to TB programme managers, because of affordable mobile electronic devices in many settings. The potential of these technologies to support patient-centred interventions, a key element of the End TB Strategy, has been recognised in recent years by the World Health Organization (WHO) [16-18].

Digital interventions are gradually being integrated into practice, and are tested and evaluated in field trials focused specifically on TB prevention and care [19]. SMS and other technologies which can communicate via cellular or internet networks, such as video-observed therapy (VOT) and the Medication Event Monitoring System and other electronic medication monitors (MMs), are being employed. For example, VOT was first used for TB in 2007 in several clinical programmes in London, UK, as an alternative to in-person treatment observation [20]; it has also been evaluated in studies in Australia, the USA and Mexico [21-23].

Although several evaluations involving TB patients have now been completed, a comprehensive review of the role of digital technologies in improving TB care has not been performed. We reviewed the published literature to determine whether digital technologies were effective at improving TB treatment adherence, and treatment outcomes (e.g. completion, cure, loss to follow-up) as defined by the WHO [24].

\section{Methods}

Cochrane guidelines for systematic reviews of interventions were followed [25].

\section{Search strategy}

In July 2016, using search terms to denote TB disease, digital technologies and TB treatment outcomes, we searched for relevant studies from the following bibliographical databases: MEDLINE/PubMed, EMBASE, Cochrane Library of Trials, and Web of Science, as well as clinicaltrials.gov and Journal of Global Health, Journal of Telemedicine and Telecare and the Journal of Telemedicine and e-Health. WHO databases and reports were also searched. Given the dearth of published studies, we included unpublished literature when 
full results had been provided to the WHO and were available from its databases. The complete list of search terms is provided in supplementary table S1. We set no restrictions in terms of language or publication dates.

\section{Study selection; inclusion and exclusion criteria}

After the preliminary search, two authors (B.K. Ngwatu and N.P. Nsengiyumva) independently screened all titles and abstracts for eligibility. Upon initial review, any studies not related to digital health interventions for TB treatment support were excluded.

We then conducted a detailed review of the remaining abstracts and full text articles. Specifically, we included studies that reported on digital health technology interventions supporting active TB treatment, such as SMS reminders, VOT and MMs, and compared them to the local standard of care as defined by the authors, with no additional specific intervention.

We included randomised controlled trials (RCTs) and observational studies, if they had a control group and reported the effect of digital technologies on adherence to treatment or appointments, and/or any of the following standard TB treatment outcomes: cure, treatment success (cure or treatment completed) [24], loss to follow-up from treatment, or a combination of unfavourable outcomes, including death, failure and/or loss to follow-up.

We excluded studies that did not report an estimate of effect related to the intervention, and those that did not report a study end-point of relevance to our review's objective (i.e. those that only reported TB incidence, number of secondary $\mathrm{TB}$ cases, costs for patients and services, patient acceptability and feasibility). We excluded reports that only described study protocols, those where only an abstract was available, letters, editorials and position papers. If the same study was reported in different publications, we considered only the most recent publication.

\section{Data extraction}

Two reviewers (B.K. Ngwatu and N.P. Nsengiyumva) extracted data from relevant articles into a standardised template, with the following information: authors, study design, method/description (title, aim, primary and secondary outcomes and duration of study), publication year, country, target population characteristics (numbers, age and sex distribution, smear status), type of digital health intervention, comparator (DOT, or no specific intervention) and measures of effect (means ratios, risk ratios, or odds ratios with respective confidence intervals). Data were then reviewed and discussed with additional reviewers (O. Oxlade, K. Schwartzman, D. Falzon and B. Mappin-Kasirer).

\section{Quality assessment of studies}

We assessed the risk of bias in the RCTs using the Cochrane risk-of-bias 2.0 (RoB 2.0) tool [26] and reported on selection bias, performance bias, detection bias, attrition bias and reporting bias. For each feature of interest, three scores were used: low risk, high risk and unclear risk.

Selection bias in RCTs was assessed by examining whether proper sequence generation and concealment of allocation sequence were carried out. Performance bias was evaluated by reviewing the blinding of participants and/or investigators administering the interventions. Detection/outcome measurement bias was assessed by reviewing who was aware of the intervention received by study participants. Bias due to attrition or incomplete or missing outcome data was evaluated by looking at whether outcome data was available for all, or nearly all, participants. Reporting bias was assessed by the extent to which the authors appeared to report their results in the way they had originally proposed in their protocols.

For observational studies, we adapted the ROBINS-I (risk of bias in non-randomised studies of interventions) tool [27] to assess selection bias, bias due to confounding, bias due to missing data, and bias in measurement of outcomes. We used the same scoring system as for RCTs.

\section{Results}

Study selection

Our search initially identified 342 studies (figure 1). Screening of titles yielded 57 (17\%) relevant studies after eliminating 24 duplicates, 33 studies were retained.

\section{Excluded studies}

After screening the 33 abstracts, nine studies (27\%) were excluded: two study protocols, two systematic reviews, three ongoing studies, and two feasibility studies (a microchip system with an ingestible sensor, and a voice call based reminder system for adherence). 


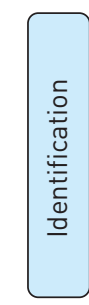

Records identified through database
searching $(n=342)$

Relevant study titles retained $(n=46]$
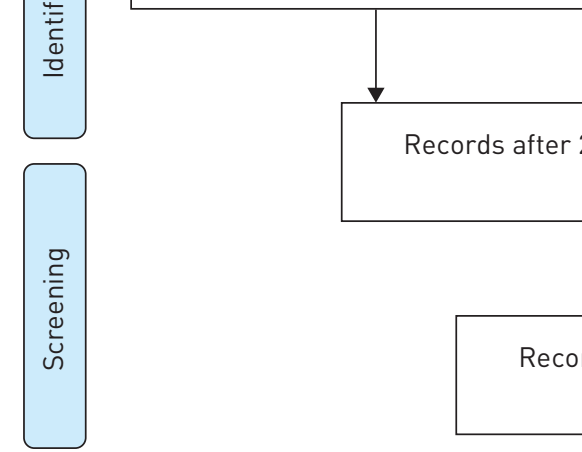

Records after 24 duplicates removed ( $n=33$ )
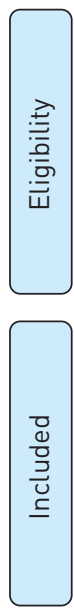

SEIERE 1 PRISMA selection of studies.

After full-text review of the 24 articles, 17 (71\%) were excluded: seven used end-points that differed from our primary end-points of interest, three had no control group, four reported only qualitative outcomes, two focused only on costs, and one was an ongoing study.

Details of the excluded studies are provided in supplementary table S2.

\section{Included studies}

Ultimately, seven studies were retained: three observational studies and four RCTs. All seven had a control group and provided estimates of effect: one evaluated MMs [28], three evaluated SMS reminders [29-31], two examined VOT [22, 23] and one examined the combination of SMS and MM [32]. The studies were carried out in seven geographically diverse settings: Argentina, Australia, Cameroon, China, Pakistan, South Africa and the USA [22, 23, 28-32]. Details of these seven studies, including details of the intervention evaluated, are listed in table 1.

\section{Findings}

Detailed results for each intervention studied are shown in table 2. Treatment adherence is reported as described in the articles. Most often it was based on the number of expected doses actually taken, but sometimes on the retention of patients during the study period.

MM

A South African pilot study reported a risk ratio of 2.3 (95\% CI 1.6-3.4) [28] for improvement in cure rates, but the comparison group was a historical, non-concurrent control group. This study also suggested significant improvement in smear conversion at 2 months, with risk ratio 1.62 (95\% CI 1.09-2.42), although again the control group was historical (non-concurrent). A larger RCT in China reported a statistically 
TABLE 1 Details of studies included in this systematic literature review

\begin{tabular}{|c|c|c|c|c|c|}
\hline Digital intervention & Objective & $\begin{array}{l}\text { Method/ study } \\
\text { design/ location }\end{array}$ & Subjects n & Intervention & $\begin{array}{l}\text { Standard of care } \\
\text { in control arm }\end{array}$ \\
\hline
\end{tabular}

\section{Electronic medication monitors}

BRoomheAD [28]

To evaluate cost
implications and
health outcomes of
the implementation of
SIMPill, in new
smear-positive TB
patients receiving TB
medication

\section{SMS reminders}

Bediang [31]

IRIBARREN [29]

Mohammed [30]

\section{Video-observed therapy}

CHuck [22]

WADE [23]
To evaluate the reminders as an adjunct to DOT in improving TB treatment adherence and success.

To evaluate the acceptance and feasibility of a patient-based text intervention to promote their adherence to TB treatment

To measure the impact of a two-way SMS reminder system

on TB treatment outcomes

\footnotetext{
To determine whether video technology for remote observation of patients on anti-TB treatment (VOT) is as effective as in-person DOT

To assess the
effectiveness related
to patient
compliance, cost
effectiveness,
acceptability and
sustainability of
video-based DOT
}
effectiveness of SMS

\begin{abstract}
A single-arm trial, with retrospective analysis of data from TB patients (historic controls) at a clinic in Northern Cape Province, South Africa
\end{abstract}

\section{Randomised,} concealed,

single-blinded controlled trial conducted at 12 TB treatment centres in Yaoundé, Cameroon

Randomised, concealed non-blinded controlled trial, conducted within a specialised hospital in Buenos Aires, Argentina

Randomised, non-blinded controlled trial at TB treatment facilities in Karachi, Pakistan

Prospective cohort study

390 patients using DOT for TB

treatment support, New York, USA

A retrospective cohort design was used, recipients of VOT were compared to in-person-DOT recipients using data at a facility in Adelaide, South Australia
120 participants (24 intervention, 96

in historic control arm), new smear-positive TB

279 patients with active TB (137 in intervention group, 142 in control groupl

37 newly diagnosed TB patients (18 in intervention group. 19 in control group)

$$
\begin{gathered}
2207 \text { newly } \\
\text { diagnosed TB } \\
\text { patients (1110 in } \\
\text { intervention arm, } \\
1097 \text { in control } \\
\text { arm) }
\end{gathered}
$$

61 patients (16\%) were assigned to VOT and 329 (84\%) to in person DOT

128 patients with active TB at the community nursing service (58 in intervention group, 70 in control group)
SIMPill a pillbox which,

DOT" when opened, sends an SMS to a server, indicating that the patient has taken their medication

One-way SMS; daily

reminders for TB

medication as adjunct to DOT

Two-way SMS; patients were instructed to SMS the clinic after self-administration of medication as a proxy of adherence (they received reminders if they did not send a message)

Two-way SMS; daily automated SMS reminders sent at prescheduled time Patient responds back via SMS or phone call

VOT worker and patient pre-arranged a schedule for the VOT calls

VOT; patients received

DOT

SAT

DOT

DOT daily video calls from the facility 


\begin{tabular}{lcccc} 
TABLE 1 Continued & Objective & $\begin{array}{c}\text { Method/study } \\
\text { design/location }\end{array}$ & Subjects $n$ & $\begin{array}{c}\text { Intervention } \\
\text { Digital intervention }\end{array}$ \\
\hline
\end{tabular}

\section{SMS reminders, medication/ electronic monitors levaluated alone or in combination]} LIU [32]

\author{
To evaluate the \\ effectiveness of text \\ messaging and \\ medication monitors \\ in improving TB \\ medication \\ adherence
}

$\begin{array}{cc}\text { Cluster randomised } & 4173 \text { TB patients } \\ \text { trial lusing } & \text { (1104 control, 1008 } \\ \text { stratification and } & \text { SMS arm, 997 MM } \\ \text { restriction) } & \text { arm, 1064 } \\ \text { conducted in four } & \text { combined SMS and } \\ \text { provinces in China } & \text { MM) }\end{array}$

\author{
Two-way SMS, MM or \\ combination of two-way \\ SMS and MM reminders \\ on dose days to take \\ medicine and to attend \\ follow-up visits \\ Physicians were advised \\ to provide more active \\ follow-up to patients \\ with adherence issues
}

\author{
$\mathrm{DOT}^{\#}$ and $\mathrm{MM}$ \\ without reminders
}

TB: tuberculosis; MM: medication monitor; SMS: short message service; DOT: directly observed treatment; SAT: self-administered treatment. \#: patients on in-person DOT were defined as those who had doses of medication observed at a health department or hospital clinic or in the community, and did not receive the intervention being evaluated in the study. Depending on the study, some treatment doses in the "DOT" arm were, in fact, self-administered.

significant effect on adherence with a MM relative to standard care, with an adjusted means ratio of 0.58 for percentage of patient-months where at least $20 \%$ of doses were missed (95\% CI 0.42-0.79) [32].

SMS

Four RCTs [29-32] evaluating SMS as medication reminders showed no statistically significant effect on treatment completion, when compared with the local standard of TB care. In three of these [23, 24, 26], the risk ratios for completion, success or cure ranged from 1.0-1.45, and the 95\% CI fell within these values in all three trials. In the SMS arm of the fourth trial [25], compared to standard care the adjusted risk ratio for failure, death, and loss to follow-up combined was 0.44 (95\% CI $0.17-1.13$ ). In the same four studies, SMS reminders were found to have very little to no, impact on TB medication doses taken, with risk or means ratios approaching 1 . Three of the four studies [23-25] employed two-way text messaging between patients and providers, while one study used one-way text reminders to patients [26].

VOT

One study in New York City (NY, USA) reported a risk ratio of 0.99 (95\% CI 0.93-1.05) for treatment completion with VOT compared with in-person DOT, although it also reported a positive effect on appointment attendance (95\% adherence to scheduled VOT sessions compared to $91 \%$ of scheduled DOT visits) [22]. Another study in Australia reported a higher proportion of observed treatment doses with VOT compared to in-person DOT, but the effect on treatment completion rates was not statistically significant [23]. In both studies, VOT substantially reduced the personnel time needed for treatment supervision. These studies used synchronous (videoconferencing) technology, which might have a different effect on adherence compared with asynchronous (recorded) VOT [21].

\section{SMS and MM}

The RCT in China [25] included an additional arm that combined both two-way SMS and MM used as medication dose reminders; there was potential synergy in that the adjusted means ratio for percentage of patient-months where at least $20 \%$ of doses were missed was 0.49 (95\% CI $0.27-0.88$ ). In that study, $14 \%$ of participants in the combined intervention arm missed $20 \%$ or more doses, compared to $30 \%$ in the control arm [32].

\section{Assessment of methodological quality}

Quality assessment and risk of bias in the randomised trials reviewed are shown in table 3. We found low selection bias, as all publications of randomised trials provided information about the processes of random sequence generation and/or allocation concealment in the studies. Overall, there was high bias of performance across the trials. Detection/outcome measurement bias was high for one trial since the 
TABLE 2 Impact of digital health interventions on study outcomes: intervention versus control groups

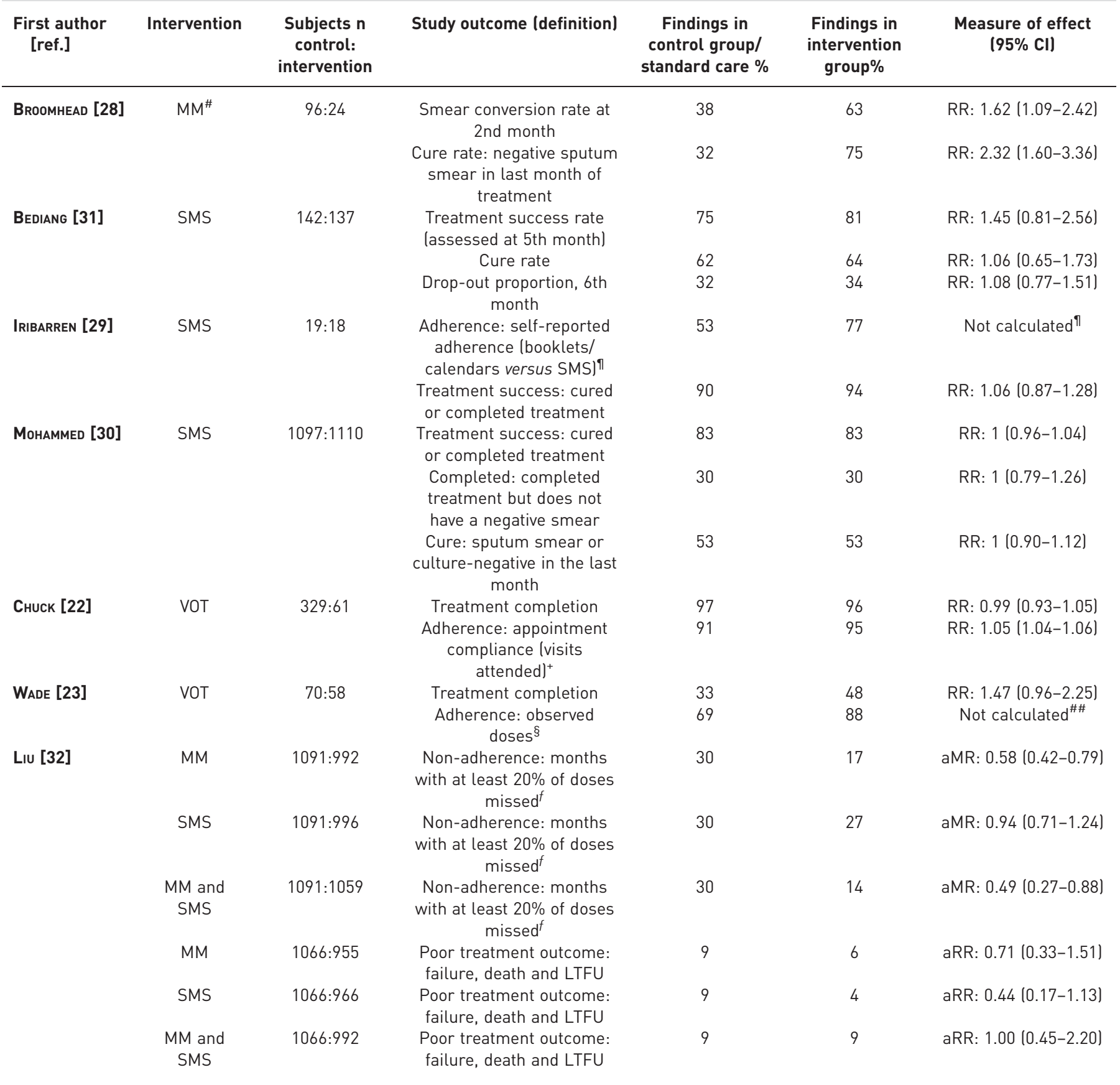

MM: medication monitor; RR: risk ratio; SMS: short message service; VOT: video-observed therapy; aMR: adjusted means ratio; LTFU: loss to follow-up; aRR: adjusted risk ratio. " : historical control; " : adherence comparison between the intervention and control groups is hampered by the fact that $47 \%$ of the calendars were not returned for analysis; ${ }^{+}: 3292$ (95\%) out of 3455 sessions scheduled for patients on VOT were successfully attended, compared to $32204(91 \%)$ out of 35442 among patients on in-person DOT; §: calculated using data provided in original text articles; ${ }^{f}$ : mean of the percentage of patient-months on TB treatment where at least $20 \%$ of doses were missed; ${ }^{\# \#}$ : could not be calculated, as no risk ratios were reported in the article and absolute numbers were not provided.

assessors were aware of the intervention received by study participants, while in the remaining three trials it was unclear. Studies varied with respect to attrition bias. We found low reporting bias in three of the trials; we could not evaluate one of the trials as the trial protocol was not available. Further details of the quality assessment for the randomised trials are in supplementary table S3 and supplementary figures S1 and S2. 
TABLE 3 Quality assessment/risk of bias of randomised trials included in the review

\begin{tabular}{|c|c|c|c|c|c|}
\hline First author [ref.] & Selection bias & Performance bias & Detection bias & Attrition bias & Reporting bias \\
\hline BedIANg [31] & Low & High & High & Low & Low \\
\hline IRIBARREN [29] & Low & High & Unclear & High & Unclear \\
\hline Монамmed [30] & Low & High & Unclear & Low & Low \\
\hline LIU [32] & Low & High & Unclear & Low & Low \\
\hline
\end{tabular}

Quality assessment and risk of bias in the observational studies is shown in table 4. Selection bias could not be assessed, but there was high risk of bias due to confounding in most studies as demographic characteristics, disease severity, socioeconomic status and other confounding factors were not evaluated or accounted for. Outcome measurement bias was also high since the assessors were aware of interventions received by study participants. Bias due to missing data varied. We could not evaluate reporting bias as the study protocols were not available.

\section{Discussion}

Digital health interventions are increasingly used to support TB treatment in diverse settings globally. Studies in this review suggest that some digital interventions can potentially improve medication adherence and patient outcomes. While evidence remains incomplete, and generalisability limited, the studies reviewed suggest these technologies may be at least as effective as the standard of care. Compared with direct in-person treatment observation, VOT and MM technologies may improve efficiency, save money and reduce burden on patients and healthcare workers. VOT used for treatment observation in London, UK, led to improved communication between patients and providers [20,33]. Pilot single-arm VOT studies in the USA, Mexico and Belarus suggested that treatment outcomes were comparable to those with in-person DOT, with markedly reduced health system costs [21,34]. These studies compared VOT to high-functioning DOT programmes; there was no difference in adherence, which suggests that comparably high adherence can be obtained using digital technologies for treatment support. In settings where digital solutions cost less and/or are easier to implement and use than the standard of care, VOT may be a beneficial alternative. Along similar lines, a pilot study of MM use in South Africa [28] suggested substantial return on investment through improved treatment outcomes and cost savings.

SMS studies in this review showed no significant effect on clinical treatment outcomes and adherence, though there was weak evidence of an impact on loss to follow-up during treatment. Results from three large RCTs of one- or two-way SMS during TB treatment [30-32] contrast with evidence from meta-analyses of RCTs in other disease settings. For example, studies have shown a positive effect of two-way SMS on HIV treatment adherence and treatment outcomes [35-37]. One potential explanation for this difference is that SMS communication and support add more to self-administered treatment (as is the case for HIV) than to direct observation and in-person treatment support, as is done in well-functioning TB programmes.

More generally, where DOT is systematically well delivered, digital technologies such as VOT and MMs are unlikely to improve adherence or clinical outcomes, but can substantially bolster efficiency and convenience for patients and providers. In TB treatment contexts where DOT is not effectively delivered, digital technologies offer the possibility of improving adherence and clinical outcomes, though suitable evidence is needed. An important example is latent TB infection, where most treatment is currently self-administered.

The application of mobile phone interventions to help TB efforts continues to generate interest. After we completed this systematic review, two RCTs were completed. A study of SMS reminders used among TB

\begin{tabular}{|c|c|c|c|c|c|}
\hline $\begin{array}{l}\text { First author } \\
\text { [ref.] }\end{array}$ & $\begin{array}{l}\text { Selection } \\
\text { bias }\end{array}$ & Confounding & $\begin{array}{c}\text { Measurement of } \\
\text { outcome }\end{array}$ & $\begin{array}{l}\text { Missing } \\
\text { data }\end{array}$ & $\begin{array}{l}\text { Reporting } \\
\text { bias }\end{array}$ \\
\hline BROOMHEAD [28] & Unclear & High & High & Low & Unclear \\
\hline Снuск [22] & Unclear & High & High & Low & Unclear \\
\hline W $_{A D E}[23]$ & Unclear & High & High & Unclear & Unclear \\
\hline
\end{tabular}


patients in China described improved treatment completion and fewer missed doses with daily one-way SMS reminders [38]. The results of another RCT, in Kenya, investigating treatment support and supervision built around daily two-way SMS communication, are not yet published, but the protocol is available online [39].

Until recently, the evidence base underpinning the use of digital interventions to support TB care has relied largely on experience in other disease contexts. Although a systematic review of studies examining the effect of SMS technology on TB treatment adherence was published in 2013 [40], our systematic review, to our knowledge, is the first to consider studies evaluating the effect of several digital health technologies on TB treatment adherence and treatment outcomes. Since 2015, the World Health Organization (WHO), the European Respiratory Society, and other leading technical and funding partners have developed target product profiles for digital health interventions to help steward the implementation and evolution of these potentially useful tools [19]. The latest WHO TB treatment guidelines highlight the potential contributions of SMS, VOT and MMs in supporting adherence and treatment delivery for patients and programmes [18]. This policy now needs to be complemented by additional evidence as well as by pragmatic implementation guidance, to enable users to translate research findings into local practice.

Our review has several limitations. We focused on quantitative comparisons of adherence and clinical outcomes, as these are fundamental to the evidence base. For this reason, we have not provided a detailed review of studies which focused exclusively on cost, feasibility, acceptability and/or qualitative assessments. However, these are also essential in considering introduction and scale-up of such technologies, and in supplementary table S2, we provide suitable references to such studies.

Despite an extensive search, we only found seven relevant reports. Given the marked heterogeneity of study designs, end-points and settings, we were unable to pool the estimates of effect, and could only summarise findings as reported from each of the seven studies. It was difficult to fully characterise the methodological quality of some studies, as the necessary information was often incompletely reported. This should be kept in mind when interpreting study results. Finally, a recurrent limitation of the existing literature is the emphasis on short-term measures of medication adherence, e.g. missed doses. The relationship of such measures to relevant clinical outcomes for patients and providers is not always certain.

With the growing interest in mobile technologies and the increasing affordability and global expansion of broadband internet and mobile phones [11], further evaluation of digital health interventions is urgently needed - ideally in adequately powered RCTs, or at a minimum, in observational studies with adequate sample size, suitable controls, and appropriate TB treatment outcomes as study end-points. Practices such as registering and publishing study protocols a priori, and using reporting guidelines such as CONSORT (consolidated standards for reporting trials) for RCTs, can improve the quality of the evidence. Currently there are several ongoing or unpublished RCTs evaluating clinical outcomes of TB treatment which apply these technologies in diverse settings; their protocols are available online [31, 33, 39, 41-43]. Beyond RCTs, further research on implementation is needed to evaluate and understand the feasibility, acceptability and cost of the various digital health interventions in diverse communities and locations. It will be relevant to explore the possible influence of digital technologies on behaviour change at other points on the patient pathway - for example, in preventing initial loss-to-follow-up before TB treatment initiation, or in detecting and managing adverse events during treatment [44].

In conclusion, the evidence base from studies on digital technologies targeting $\mathrm{TB}$ is slowly growing. Despite interest in the use of digital technologies to improve the care of persons with $\mathrm{TB}$, their reported impact has been variable and evidence from implementation studies remains sparse. Our findings suggest that certain digital technologies can support TB treatment efforts while reducing both patient and provider costs, as well as patient inconvenience. Worldwide, patients and providers are increasingly using mobile devices to communicate. It is thus important to understand how technologies could best be used to provide better patient-centred treatment support and to allocate resources more judiciously. Data from ongoing and future research, including non-inferiority studies, pragmatic trials and cost-effectiveness analyses, will aid in the optimisation of practical approaches. These could include targeting different entry points on the behaviour change pathway which have not yet been explored, and combining several technologies - either sequentially or simultaneously - to better leverage their effects on TB treatment adherence, and ultimately, clinical outcomes.

\section{Acknowledgements}

The members of the Collaborative Group on the Impact of Digital Technologies on TB are as follows.

I. Abubakar (University College London, London, UK); N. Alipanah (University of California, San Francisco, CA, USA); M. Bastos (Rio de Janeiro State University, Rio de Janeiro, Brazil); D. Boccia (TB Centre, London School of Hygiene \& Tropical Medicine, London, UK); D. Chin (Bill \& Melinda Gates Foundation, Seattle, WA, USA); T. Cohen (Yale University, New Haven, CT, USA); J.L. Davis ${ }^{8}$ (Yale University, New Haven, CT, USA); C. Denkinger (FIND, 
Geneva, Switzerland); D. Falzon (Global TB Programme, WHO, Geneva, Switzerland); K. Fielding (TB Centre, London School of Hygiene \& Tropical Medicine, London, UK); G. Fox (University of Sydney, Sydney, Australia); C. Free (TB Centre, London School of Hygiene \& Tropical Medicine, London, UK); R.S. Garfein (University of California San Diego, San Diego, CA, USA); A. Hayward (University College London, London, UK); E. Jaramillo (Global TB Programme, WHO, Geneva, Switzerland); R. Lester (University of British Columbia, Vancouver, BC, Canada); J. Lewis (TB Centre, London School of Hygiene \& Tropical Medicine, London, UK); B. Mappin-Kasirer (Montreal Chest Institute, Respiratory Epidemiology and Clinical Research Unit, Montreal, QC, Canada); F. Marx (Stellenbosch University, Cape Town, South Africa); D. Menzies (Montreal Chest Institute, Respiratory Epidemiology and Clinical Research Unit, Montreal, QC, Canada); G.B. Migliori (WHO Collaborating Centre for TB and Lung Diseases, Maugeri Care and Research Institute, Tradate, Italy); P. Nahid (University of California San Francisco, San Francisco, CA, USA); B. Ngwatu (Global TB Programme, WHO, Geneva, Switzerland); N.P. Nsengiyumva (Montreal Chest Institute, Respiratory Epidemiology and Clinical Research Unit, Montreal, QC, Canada); N.L. Nguyen (Global TB Programme, WHO, Geneva, Switzerland); O. Oxlade (Montreal Chest Institute, Respiratory Epidemiology and Clinical Research Unit, Montreal, QC, Canada); K. Schwartzman (Montreal Chest Institute, Respiratory Epidemiology and Clinical Research Unit, Montreal, QC, Canada); K. Siddiqi (University of York, York, UK); A. Story (University College London, London, UK); B. Thomas (The Arcady Group, Richmond, VA, USA); A. Trajman (Rio de Janeiro State University, Rio de Janeiro, Brazil); M. Yassin (The Global Fund to Fight AIDS, Tuberculosis and Malaria, Geneva, Switzerland).

All authors contributed to the ideation and writing of this manuscript, and agree with the conclusions drawn. D. Falzon, E. Jaramillo and N.L. Nguyen are staff members of WHO; they alone are responsible for the views expressed in this publication and they do not necessarily represent the decisions or policies of WHO. The designations used and the presentation of the material in this publication do not imply the expression of any opinion whatsoever on the part of WHO concerning the legal status of any country, territory, city or area, or of its authorities, or concerning the delimitation of its frontiers or boundaries.

\section{References}

1 Roine R, Ohinmaa A, Hailey D. Assessing telemedicine: a systematic review of the literature. CMAJ 2001; 165: 765-771.

2 Goldfarb NM. Improving subject compliance with smart pill bottles. J Clin Res Best Pract 2007; 3: 1-5.

3 Vervloet M, van Dijk L, Santen-Reestman J, et al. SMS reminders improve adherence to oral medication in type 2 diabetes patients who are real time electronically monitored. Int J Med Inform 2012; 81: 594-604.

4 Checchi KD, Huybrechts KF, Avorn J, et al. Electronic medication packaging devices and medication adherence: a systematic review. JAMA 2014; 312: 1237-1247.

5 Verhoeven F, Tanja-Dijkstra K, Nijland N, et al. Asynchronous and synchronous teleconsultation for diabetes care: a systematic literature review. J Diabetes Sci Technol 2010; 4: 666-684.

6 Verhoeven F, van Gemert-Pijnen L, Dijkstra K, et al. The contribution of teleconsultation and videoconferencing to diabetes care: a systematic literature review. J Med Internet Res 2007; 9: e37.

7 Krishna S, Boren SA. Diabetes self-management care via cell phone: a systematic review. J Diabetes Sci Technol 2008; 2: 509-517.

8 Gentry S, van-Velthoven MH, Tudor Car L, et al. Telephone delivered interventions for reducing morbidity and mortality in people with HIV infection. Cochrane Database Syst Rev 2013; 5: CD009189.

9 Free C, Phillips G, Galli L, et al. The effectiveness of mobile-health technology-based health behaviour change or disease management interventions for health care consumers: a systematic review. PLoS Med 2013; 10: e1001362.

10 Vodopivec-Jamsek V, de Jongh T, Gurol-Urganci I, et al. Mobile phone messaging for preventive health care. Cochrane Database Syst Rev 2012; 12: CD007459.

11 Ambia J, Mandala J. A systematic review of interventions to improve prevention of mother-to-child HIV transmission service delivery and promote retention. J Int AIDS Soc 2016; 19: 20309.

12 Castaño PM, Bynum JY, Andrés R, et al. Effect of daily text messages on oral contraceptive continuation: a randomized controlled trial. Obstet Gynecol 2012; 119: 14-20.

13 Lund S, Hemed M, Nielsen BB, et al. Mobile phones as a health communication tool to improve skilled attendance at delivery in Zanzibar: a cluster-randomised controlled trial. BJOG 2012; 119: 1256-1264.

14 World Health Organization. Global tuberculosis report 2016. http://apps.who.int/iris/bitstream/10665/250441/1/ 9789241565394-eng.pdf Date last accessed: November 14, 2017.

15 Karumbi J, Garner P. Directly observed therapy for treating tuberculosis. Cochrane Database Syst Rev 2015; 29: CD000343.

16 World Health Organization. Digital health for the End TB Strategy: an agenda for action. Geneva, World Health Organisation, 2015 www.who.int/tb/areas-of-work/digital-health/Digital_health_EndTBstrategy.pdf?ua=1 Date last accessed: November 14, 2017.

17 World Health Organization. Towards TB elimination: an action framework for low-incidence countries: World Health Organization. http://apps.who.int/iris/bitstream/10665/132231/1/9789241507707_eng.pdf Date last accessed: November 14, 2017.

18 World Health Organization. Guidelines for treatment of drug-susceptible tuberculosis and patient care, 2017 update. http://apps.who.int/iris/bitstream/10665/255052/1/9789241550000-eng.pdf Date last accessed: November 14, 2017.

19 Falzon D, Timimi H, Kurosinski P, et al. Digital health for the End TB Strategy: developing priority products and making them work. Eur Respir J 2016; 48: 29-45.

20 Story A, Garfein RS, Hayward A, et al. Monitoring therapy compliance of tuberculosis patients by using video-enabled electronic devices. Emerging Infect Dis 2016; 22: 538-540.

21 Garfein R, Collins K, Munoz F, et al. Feasibility of tuberculosis treatment monitoring by video directly observed therapy: a binational pilot study. Int J Tuberc Lung Dis 2015; 19: 1057-1064.

22 Chuck C, Robinson E, Macaraig M, et al. Enhancing management of tuberculosis treatment with video directly observed therapy in New York City. Int J Tuberc Lung Dis 2016; 20: 588-593.

23 Wade VA, Karnon J, Eliott JA, et al. Home videophones improve direct observation in tuberculosis treatment: a mixed methods evaluation. PLoS one 2012; 7: e50155. 
24 World Health Organization. Definitions and reporting framework for tuberculosis - 2013 revision. http://apps. who.int/iris/bitstream/10665/79199/1/9789241505345_eng.pdf Date last accessed: November 14, 2017.

25 Higgins JP, Green S. Cochrane handbook for systematic reviews of interventions. Hoboken, New Jersey, USA, John Wiley \& Sons, 2011.

26 Higgins JPT, Sterne JAC, Savović J, et al. A revised tool for assessing risk of bias in randomized trials. In: J Chandler, J McKenzie, I Boutron, eds. Cochrane Methods. London, Cochrane Database of Systemic Reviews, 2016; Suppl. 1.

27 Sterne J, Hernán MA, Reeves BC, et al. ROBINS-I: a tool for assessing risk of bias in non-randomised studies of interventions. BMJ 2016; 355: i4919.

28 Broomhead S, Mars M. Retrospective return on investment analysis of an electronic treatment adherence device piloted in the Northern Cape Province. Telemed J E Health 2012; 18: 24-31.

29 Iribarren S, Beck S, Pearce PF, et al. TextTB: A mixed method pilot study evaluating acceptance, feasibility, and exploring initial efficacy of a text messaging intervention to support TB treatment adherence. Tuberc Res Treat 2013; 2013: 12 .

30 Mohammed S, Glennerster R, Khan AJ. Impact of a daily SMS medication reminder system on tuberculosis treatment outcomes: a randomized controlled trial. PloS one 2016; 11: e0162944.

31 Bediang G, Stoll B, Elia N, et al. SMS reminders to improve tuberculosis cure in developing countries (TB-SMS Cameroon): a protocol of a randomised control study. Trials 2014; 15: 35. Date last accessed: December 15, 2017.

32 Liu X, Lewis JJ, Zhang $\mathrm{H}$, et al. Effectiveness of electronic reminders to improve medication adherence in tuberculosis patients: a cluster-randomised trial. PLoS Med 2015; 12: e1001876.

33 Hayward A, Garber E. TB Reach 5: to compare the efficacy of video observed treatment (VOT) versus directly observed treatment (DOT) in supporting adherence in patients with active tuberculosis. ISRCTN Reg 2014; https:// doi.org/10.1186/ISRCTN26184967.

34 Sinkou H, Hurevich $\mathrm{H}$, Rusovich V, et al. Video-observed treatment for tuberculosis patients in Belarus: findings from the first programmatic experience. Eur Respir J 2017; 49: 1602049.

35 Wald DS, Butt S, Bestwick JP. One-way versus two-way text messaging on improving medication adherence: metaanalysis of randomized trials. Am J Med 2015; 128: 1139.

36 Mbuagbaw L, van der Kop ML, Lester RT, et al. Mobile phone text messages for improving adherence to antiretroviral therapy (ART): an individual patient data meta-analysis of randomised trials. BMJ Open 2013; 3: e00395.

37 Finitsis DJ, Pellowski JA, Johnson BT. Text message intervention designs to promote adherence to antiretroviral therapy (ART): a meta-analysis of randomized controlled trials. PloS one 2014; 9: e88166.

38 Fang X-H, Guan S-Y, Tang L, et al. Effect of short message service on management of pulmonary tuberculosis patients in Anhui Province, China: a prospective, randomized, controlled study. Med Sci Monit 2017; 23: 2465-2469.

39 Self-verification and support via mobile phones drastically improves tuberculosis treatment success in LMIC settings. https://clinicaltrials.gov/ct2/show/record/NCT03135366 Date last updated: Aug 25, 2017. Date last accessed: November 14, 2017.

40 Nglazi MD, Bekker L-G, Wood R, et al. Mobile phone text messaging for promoting adherence to antituberculosis treatment: a systematic review. BMC Infect Dis 2013; 13: 566.

41 Virtually observed treatment (VOT) for tuberculosis patients in Moldova. https://clinicaltrials.gov/ct2/show/ NCT03266003 Date last updated: September 25, 2015. Date last accessed: November 14, 2017.

42 Liu X. A trial of an electronic pill box with reminders for patients taking treatment for tuberculosis. ISRCTN Reg 2016; https://doi.org/10.1186/ISRCTN35812455.

43 An evaluation of traditional directly observed therapy (DOT) and electronic DOT for TB treatment. https:// clinicaltrials.gov/ct2/show/NCT03266003 Date last updated: August 29, 2017. Date last accessed: November 14, 2017.

44 Mehta U, Durrheim DN, Blockman M, et al. Adverse drug reactions in adult medical inpatients in a South African hospital serving a community with a high HIV/AIDS prevalence: prospective observational study. $\mathrm{Br}$ Clin Pharmacol 2008; 65: 396-406. 